\title{
MicroRNA-195 is associated with regulating the pathophysiologic process of human laryngeal squamous cell carcinoma
}

\author{
HAIFENG PANG, XUEMEI XU, LINLIN DAI, KUN WANG and XIANYI YAO \\ Department of Otolaryngology, The Fourth Affiliated Hospital of \\ Harbin Medical University, Harbin, Heilongjiang 150001, P.R. China
}

Received January 13, 2016; Accepted December 20, 2017

DOI: $10.3892 / \mathrm{mmr} .2018 .8523$

\begin{abstract}
MicroRNAs (miRNAs) have been reported to be associated with the modulation of tumor development, including alterations associated with the development of human laryngeal squamous cell carcinoma (LSCC). The present study was designed to investigate whether miRNA-195 was associated with the pathophysiologic process of human LSCC and to identify its potential roles and underlying molecular mechanisms. To determine whether miRNA-195 serves a role in LSCC, reverse transcription-quantitative polymerase chain reaction was used to detect miRNA-195 expression in LSCC tissues. The tumor-suppressive effect of miRNA-195 was determined by in vitro assays. Gain-of-function studies using miRNA-195 mimics were performed to investigate cell viability, migration and invasion, and apoptosis in the AMC-HN-8 cell line. Western blotting was performed to reveal the molecular mechanisms of miRNA-195 and its downstream signaling pathways in the LSCC AMC-HN-8 cell line. The present study demonstrated that miRNA-195 is downregulated in primary LSCC tumors. Upregulating miRNA-195 in vitro suppressed cell viability, migration and invasion in AMC-HN-8 cells. Overexpression of miRNA-195 alone in AMC-HN-8 cells was sufficient to induce cell apoptosis, as identified by terminal deoxynucleotidyl transferase dUTP nick end labeling assay. Compared with the high expression of miRNA-195 in AMC-HN-8 cells, the expression levels of vascular endothelial growth factor receptor-II protein and downstream signaling pathway proteins, which were associated with cell viability, migration, invasion and apoptosis, were markedly decreased compared with control or miRNA-195 negative control treatment group. Together, these data suggest the therapeutic potential of miRNA-195
\end{abstract}

Correspondence to: Dr Xianyi Yao, Department of Otolaryngology, The Fourth Affiliated Hospital of Harbin Medical University, 37 Yiyuan Street, Harbin, Heilongjiang 150001, P.R. China E-mail:xingu1a@163.com

Key words: laryngeal squamous cell carcinoma, microRNA-195, vascular endothelial growth factor receptor 2, viability, migration, invasion, apoptosis in modulating cell growth, migration and apoptosis during the pathophysiological progression of LSCC and that miRNA-195 may serve as a potential therapeutic target in human LSCC.

\section{Introduction}

Laryngeal squamous cell carcinoma (LSCC) is the second most common malignancy in the head and neck region and is reported to account for $\sim 2.4 \%$ of all new malignancies worldwide each year (1). The incidence of LSCC has been rising gradually, particularly in Northeast China (2). Although rapid progress has recently been made in treatment, the therapeutic outcomes and the overall 5-year survival rate remain unsatisfactory (3). Together with invasive and metastatic behaviors, resistance to chemotherapy has been a major obstacle in improving the 5-year survival rate of LSCC patients (4). Furthermore, total laryngectomy, the recommended treatment for advanced patients, leads to serious lung infection and the loss of speech (5). Therefore, more effective diagnostic techniques for early stage LSCC have long been warranted. The improved detection of useful biological and molecular markers in the diagnosis and therapy of LSCC, and a new strategy for the treatment of LSCC, are urgently required.

It has been suggested that conventional pathological prognostic parameters are insufficient to accurately evaluate the clinical prognosis of patients with LSCC (6). However, biomarkers are considered to be beneficial not only in evaluating the prognosis but also in guiding personalized therapy for patients with cancer (7), which further indicates the importance of identifying potential biomarkers for human LSCC. MicroRNAs (miRNAs) are a family of endogenous small single-strand RNAs with a length between 21 and 25 nucleotides, which have emerged as an important class of gene regulators (8). It has been demonstrated that miRNAs serve critical roles in tumor cell viability, differentiation, metastasis and apoptosis in vitro and in vivo (9). In addition, miRNAs have been functionally classified as tumor suppressors or proto-oncogenes and are aberrantly expressed indifferent cancers, including lung (10), liver (11), stomach (12), leukemia (13), lymphoma (14), breast (15), colorectal (16), and head and neck cancer (17). It has been suggested that miRNAs may act as the molecular targets for human cancer diagnosis 
and personalized therapy $(18,19)$. miRNA-195 has been identified to be downregulated in a variety of human solid tumors, but the potential biological roles of miRNA-195 in LSCC remain to be elucidated.

The present study initially examined the expression of miRNA-195 in LSCC and identified downregulation of miRNA-195 in LSCC cancer tissue. Subsequently, the role of miRNA-195 in cell viability, migration and apoptosis in AMC-HN-8 cells was demonstrated. It was identified that miRNA-195 modulated vascular endothelial growth factor receptor 2 (VEGFR2) expression, and was thereby associated with the VEGFR2 signaling network. These findings demonstrated that miRNA-195 serves a key role in the pathologic progression of LSCC and revealed the potential molecular mechanisms of miRNA-195 in human LSCC.

\section{Materials and methods}

Patient samples. A total of 23 cases of laryngeal squamous cell carcinoma tissue samples and adjacent healthy tissue samples between February 2016 and March 2017 were obtained from the Department of Otolaryngology in the Fourth Affiliated Hospital of Harbin Medical University (Harbin, China). There were 10 males and 13 females in the disease group, with a median age of 61 years. The present study was performed in accordance with the ethical codes of the World Medical Association (20) and was approved by the Ethics Committee for Use of Human Samples of Harbin Medical University. All human participants provided informed written consent and all clinical investigations were conducted according to the principles expressed in the Declaration of Helsinki. All specimens were coded and identified. The specimens comprised a panel of 23 LSCC patient cases obtained during surgical procedures, which were immediately stored in liquid nitrogen or fixed in formalin.

Cell culture. AMC-HN-8 cells used in the present study were purchased from American Type Culture Collection (ATCC; Manassas, VA, USA). AMC-HN-8 cells were cultured in RPMI-1640 (Gibco; Thermo Fisher Scientific, Inc., Waltham, $\mathrm{MA}, \mathrm{USA}$ ) at $37^{\circ} \mathrm{C}$ with $5 \% \mathrm{CO}_{2}$ in a humidified incubator. The cultures were supplemented with $10 \%$ fetal bovine serum (Gibco; Thermo Fisher Scientific, Inc.) and $100 \mu \mathrm{g} / \mathrm{ml}$ penicillin/streptomycin.

Oligo transfection, miRNA-195 knockdown, and miRNA-195 overexpression in AMC-HN-8 cells. Cells were seeded in antibiotic-free RPMI-1640 medium for $24 \mathrm{~h}$ prior to transfection. For upregulation of miRNA-195, the cells $\left(1 \times 10^{5}\right)$ in a six-well plate were transfected with miRNA-195 mimic (Shanghai GenePharma Co., Ltd., Shanghai, China) using Lipofectamine ${ }^{\circledR} 2000$ (Invitrogen; Thermo Fisher Scientific, Inc.) in serum-free Opti-MEM (Gibco; Thermo Fisher Scientific, Inc.) medium according to the manufacturer's instructions. For knockdown ofmiRNA-195, the cells $\left(1 \times 10^{5}\right)$ in a six-well plate were transfected with miRNA-195 inhibitor (Shanghai GenePharma Co., Ltd.) using Lipofectamine ${ }^{\circledR} 2000$ (Thermo Fisher Scientific, Inc.). Transfection complexes were added to medium at a final oligonucleotide concentration of $50 \mathrm{nM}$. miRNA-195 mimic negative control (NC) and
miRNA-195 inhibitor NC were also purchased from Shanghai GenePharma Co., Ltd. and were transfected at a final concentration of $50 \mathrm{nM}$ using Lipofectamine ${ }^{\circledR} 2000$ according to the manufacturer's instructions. Opti-MEM medium was replaced at $4 \mathrm{~h}$ post-transfection with regular culture medium and incubated at $37^{\circ} \mathrm{C}$ for a further $48 \mathrm{~h}$. The sequences of miRNA-195 mimic and mimic-NC were: 5'-UAGCAGCACAGAAAU AUUGGC-3' and 5'-UUCUCCGAACGUGUCACGUTT-3', respectively. The sequences of miRNA-195 inhibitor and inhibitor-NC were: 5'-GCCAAUAUUUCUGUGCUGCUA-3' and 5'-CAAUAUUUCUGUGCUGCUAUU-3', respectively. Following transfection, the subsequent experiments were performed within $8 \mathrm{~h}$.

Reverse transcription-quantitative polymerase chain reaction $(R T-q P C R)$. Total RNA from human LSCCs was isolated using TRIzol reagent (Thermo Fisher Scientific, Inc.) according to the manufacturer's instructions. Total RNA $(0.5 \mu \mathrm{g})$ was then reverse transcribed using the High-Capacity cDNA Reverse Transcription kit (Applied Biosystems; Thermo Fisher Scientific, Inc.) to obtain cDNA according to the manufacturer's instructions. The RNA levels of miRNA-195 were determined using Power SYBR ${ }^{\text {TM }}$ Green PCR Master Mix (Thermo Fisher Scientific, Inc.) incorporation method on an ABI 7500 fast Real Time PCR system (Applied Biosystems; Thermo Fisher Scientific, Inc.) at $95^{\circ} \mathrm{C}$ for $10 \mathrm{~min}$, followed by 40 cycles of $95^{\circ} \mathrm{C}$ for $15 \mathrm{sec}, 60^{\circ} \mathrm{C}$ for $30 \mathrm{sec}$ and $72^{\circ} \mathrm{C}$ for $30 \mathrm{sec}$, with U6 as an internal control. The relative quantitative expression of gene was calculated using method $2^{\Delta \Delta \mathrm{Cq}}(21)$. The sequences of primers were as follows: hsa-miRNA-195 forward, 5'-GGG GTAGCAGCACAGATT-3' and reverse, 5'-TCCAGTGCG TGTCGTGGA-3'; and U6 forward, 5'-GCTTCGGCACAT ATACTAAAAT-3' and reverse, 5'-CGCTTCACGAATTTG CGTGTCAT-3'.

Evaluation of cell viability via MTT assay. AMC-HN-8 cells were plated in 96-well plates and transfected with miRNA-195 mimics, miRNA-195 mimics-NC, miRNA-195 inhibitor and miRNA-195 inhibitor-NC respectively for $4 \mathrm{~h}$. Following transfection, the serum-free medium was removed and cells were cultured at $37^{\circ} \mathrm{C}$ with regular culture medium for a further $48 \mathrm{~h}$. To monitor cell survival, AMC-HN-8 cells in each well were incubated at $37^{\circ} \mathrm{C}$ for $4 \mathrm{~h}$ with $0.5 \mathrm{mg} / \mathrm{ml}$ MTT (Sigma-Aldrich; Merck KGaA, Darmstadt, Germany). Subsequently, the MTT solution was removed and the formazan crystals in the cells dissolved in $150 \mathrm{ml}$ sterile dimethyl sulfoxide (Sigma-Aldrich; Merck KGaA) by incubating at $37^{\circ} \mathrm{C}$ for $15 \mathrm{~min}$. Absorbance was recorded at $490 \mathrm{~nm}$ using an Easy Reader 340 AT (SLT-Lab Instruments, Salzburg, Austria). Relative cell survival was calculated by setting control absorbance from untreated cells at $100 \%$. Experiments were performed in triplicate.

Terminal deoxynucleotidyl transferase dUTP nick end labeling (TUNEL) assay. Apoptotic AMC-HN-8 cells in the different groups were detected using a TUNEL assay as previously described (22). TUNEL staining was detected using the In Situ Cell Death Detection kit (Roche Diagnostics GmbH, Mannheim, Germany) according to the manufacturer's instructions. Sections were also counterstained at $37^{\circ} \mathrm{C}$ for $5 \mathrm{~min}$ with 
DAPI (1:5 dilution; Invitrogen; Thermo Fisher Scientific, Inc.) for nuclei. The number of the TUNEL-positive nuclei ratio in $\geq 10$ representative microscopic fields (magnification, x200) was calculated by fluorescence microscopy (Nikon Corporation, Tokyo, Japan) to compare the apoptosis ratio within the different groups.

Invasion assays. Invasion assays were conducted using $8 \mu \mathrm{M}$ polyethylene terpthalate filters (BioCoat Matrigel Invasion Chambers; BD Pharmingen; BD Biosciences, Franklin Lakes, NJ, USA), as previously described (23). AMC-HN-8 cells $\left(5 \times 10^{4} /\right.$ well) transfected with miRNA-195 mimics, inhibitor or $\mathrm{NC}$ were allowed to invade through matrigel-coated filters for $16 \mathrm{~h}$ in a Transwell plate. The volume of medium plated in the upper chamber was $200 \mu \mathrm{l}$. Cells invaded to the lower chamber of the Transwell plate and were fixed using $4 \%(\mathrm{w} / \mathrm{v})$ paraformaldehyde at room temperature for $30 \mathrm{~min}$ and stained using $0.05 \%$ crystal violet at room temperature for $20 \mathrm{~min}$; the cell numbers were counted (magnification, x200) by a fluorescence microscopy (Nikon, Tokyo, Japan) as previously described (23).

Measurement of cell migration with Transwell migration assay. A Transwell migration assay was used to perform cell migration. Nuclepore filters with 8-nm pores (Corning, Inc., Corning, NY, USA) were coated with type IV collagen (Sigma-Aldrich; Merck KGaA) overnight at $37^{\circ} \mathrm{C}$ prior to the assay. AMC-HN-8 cells $\left(5 \times 10^{4}\right)$ in $200 \mu$ l medium (Gibco; Thermo Fisher Scientific, Inc.) from different treatment groups were added to the upper chambers, and the lower chambers were filled with normal RPMI-1640 medium (Gibco; Thermo Fisher Scientific, Inc.) supplemented with $10 \%$ fetal bovine serum (Gibco; Thermo Fisher Scientific, Inc.). Following $48 \mathrm{~h}$ of incubation at $37^{\circ} \mathrm{C}$, the cells on the upper side were removed and discarded, and the cells that migrated to the lower side of the membrane were fixed with $4 \%$ paraformaldehyde at room temperature for $30 \mathrm{~min}$, stained with $0.1 \%$ crystal violet for $30 \mathrm{~min}$ at room temperature and washed three times with PBS. The migrated cells in the lower side of the membrane were observed and imaged under an inverted microscope (magnification, x200). Images were captured from three randomly selected fields and the migrated cells counted.

Western blot analysis. The total amount of protein was extracted by RIPA buffer (Beyotime Biotechnology, Shanghai, China) from AMC-HN-8 cells for immunoblotting analysis. Briefly, the protein concentrations were determined with a bicinchoninic acid protein assay kit using bovine serum albumin (Beyotime Institute of Biotechnology) as the standard. Equal amounts of protein $(100 \mu \mathrm{g})$ were separated by $10 \%$ SDS-PAGE and blotted onto PVDF membranes (EMD Millipore, Billerica, MA, USA). The blots were blocked at room temperature with 5\% non-fat milk dissolved in PBS for $2 \mathrm{~h}$, then probed overnight at $4^{\circ} \mathrm{C}$ in $5 \%$ milk tris-buffered saline/Tween-20 with the following primary antibodies: VEGFR2 (9698S, 1:1,000; Cell Signaling Technology, Inc., Danvers, MA, USA), Raf (ab33899, 1:1,000; Abcam, Cambridge, UK), extracellular signal-regulated kinase (ERK, sc-514302, 1:1,000), phosphorylated (p)-ERK

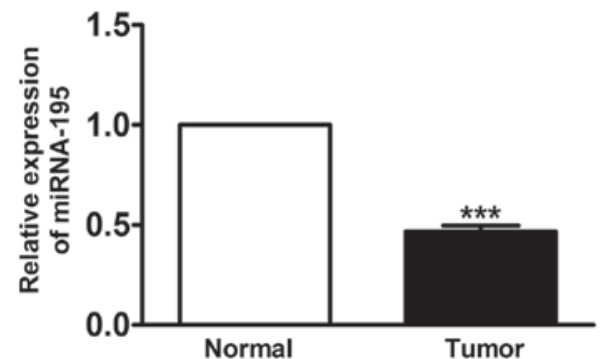

Figure 1. Expression levels of miRNA-195 in LSCC samples. Expression level of miRNA-195 in normal and LSCC samples. Data are expressed as mean \pm standard error of the mean, $n=23$. ${ }^{* * *} \mathrm{P}<0.01$ vs. normal control. miRNA, microRNA; LSCC, laryngeal squamous cell carcinoma.

(sc-81492, 1:1,000) (both from Santa Cruz Biotechnology, Inc., Dallas, TX, USA), total mitogen activated protein kinase kinase (MEK; 601121, 1:200), and p-MEK (558375, 1:1,000) (both from BD Biosciences), p-SRC (12432; 1:1,000), focal adhesion kinase (FAK; 3285; 1:1,000), p-FAK $(8556 ; 1: 1,000)$ (all from Cell Signaling Technology, Inc.), protein kinase B (AKT, 610860; 1:1,000) and p-AKT (550747; 1:1,000) (both from BD Biosciences), B-cell lymphoma 2 (Bcl-2; 15071; 1:1,000; Cell Signaling Technology, Inc.), Bcl-2-like protein 4 (Bax; 5023; 1:1,000), caspase-3 (9662; 1:500) and GAPDH (5174; 1:500) (all from Cell Signaling Technology, Inc.). Membranes were washed three times, 15 min each, with PBS containing $0.5 \%$ Tween-20 and incubated with the following secondary antibodies: Alexa Fluor ${ }^{\circledR} 700$ goat anti-mouse immunoglobulin $\mathrm{G}(\operatorname{IgG})$ [heavy and light chains $(\mathrm{H}+\mathrm{L})$; A-11029; 1:8,000] or Alexa Fluor ${ }^{\circledR} 800$ goat anti-rabbit IgG (H+L; A32730; 1:8,000; Invitrogen; Thermo Fisher Scientific, Inc.) in PBS at room temperature for $1 \mathrm{~h}$. Images of western blot bands were captured using the Odyssey Infrared Imaging System and quantified with Odyssey software, version 1.2 (both from LI-COR Biosciences, Lincoln, NE, USA) by measuring the band intensity (area $\mathrm{x}$ optical density) in each group and normalizing to GAPDH as an internal control. Unless otherwise stated, western blot analyses were repeated four times.

Statistical analysis. All quantitative data are expressed as the mean \pm standard error of the mean and analyzed using SPSS software version 13.0 (SPSS, Inc., Chicago, IL, USA). Two-tailed unpaired Student's t-tests were used between two groups and one-way analysis of variance were used for statistical evaluation of the data between multiple groups with post hoc contrasts by Student-Newman-Keuls test. $\mathrm{P}<0.05$ was considered to indicate a statistically significant difference.

\section{Results}

miRNA-195 is downregulated in human LSCC in vivo. RT-qPCR was used to determine the expression of miRNA-195 for the LSCC and the matched normal tissue samples obtained from 23 patients diagnosed with LSCC. For the LSCC samples, the mean miRNA level for miRNA-195 was significantly decreased by $55 \%$ compared with the corresponding matched samples (Fig. $1 ; \mathrm{P}<0.01$ ). 

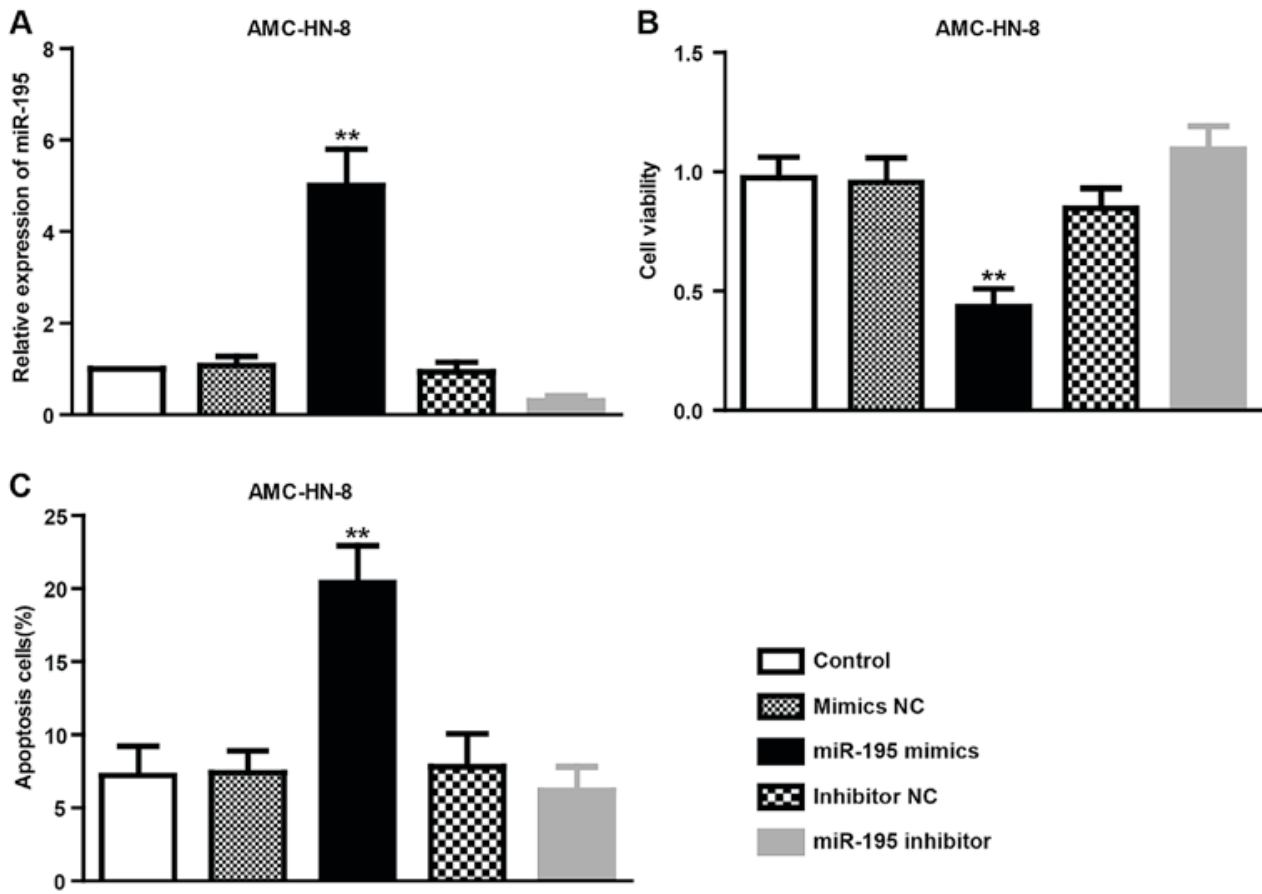

D

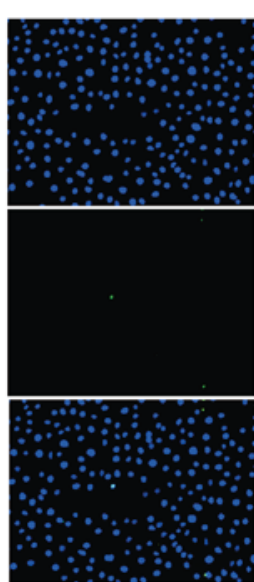

Control

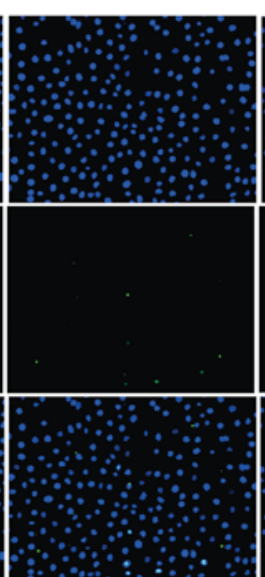

Mimics NC

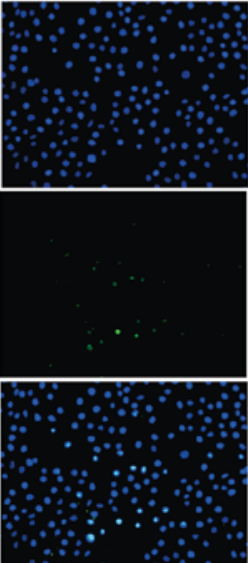

miR-195 mimics

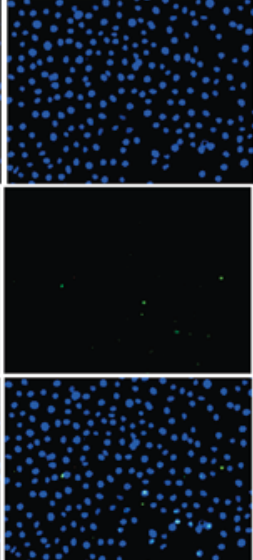

Inhibitor NC

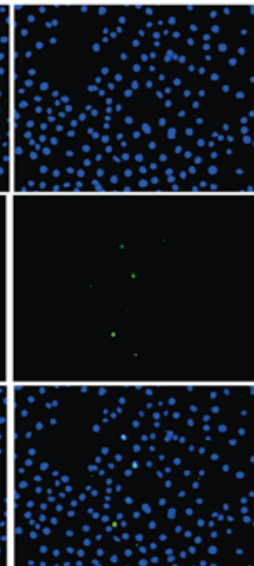

miR-195 inhibitor

Figure 2. Effects of miRNA-195 on viability and apoptosis in AMC-HN-8 cells. (A) Expression levels of miRNA-195 in AMC-HN-8 cells in different treatment groups. Data are expressed as mean \pm standard error of the mean, $\mathrm{n}=4$. (B) MTT assay for AMC-HN-8 cell viability. Data are expressed as mean \pm standard error of the mean, $\mathrm{n}=3$. (C) The number of apoptotic AMC-HN- 8 cells as determined by TUNEL assay. Data are expressed as mean \pm standard error of the mean, $\mathrm{n}=10 .{ }^{* *} \mathrm{P}<0.01$ vs. control group. (D) Apoptotic AMC-HN-8 cells determined via TUNEL staining (original magnification, $\mathrm{x} 200$ ). Green, TUNEL-stained apoptotic cell; blue, cell nucleus stained by DAPI. miRNA/miR, microRNA; NC, negative control; TUNEL, terminal deoxynucleotidyl transferase dUTP nick end labeling.

Overexpression of miRNA-195 decreases cell viability and induces apoptosis in AMC-HN-8 cells. The present study evaluated whether miRNA-195 contributes to the survival rates of AMC-HN-8 cells by overexpression with specific miRNA-195 mimics (Fig. 2). The results demonstrated that the expression level of miRNA-195 in AMC-HN-8 cells from the miRNA-195 mimics treatment group was markedly higher compared with those from the control or miRNA-195 NC treatment groups, whereas the miRNA-195 expression from inhibitor treatment was markedly lower compared with the control or NC treatment groups (Fig. 2A). In comparison with control cells, the viability of AMC-HN-8 cells as determined by MTT analysis was reduced significantly in cells treated with miRNA-195 mimics $(\mathrm{P}<0.01)$, but not with the
miRNA-195 inhibitor and scramble, which did not significantly affect the viability of AMC-HN-8 cells (Fig. 2B). In addition, TUNEL staining results demonstrated that transfection with miRNA-195 mimics, but not with the inhibitor, increased the percentage of TUNEL-positive cells in the AMC-HN-8 cell population, suggesting that miRNA-195 may provide an anti-apoptotic effect in AMC-HN-8 cells $(\mathrm{P}<0.01$; Fig. 2C and D). Together, these data suggest that upregulation of miRNA-195 may serve anti-proliferative and pro-apoptotic roles in AMC-HN-8 cells.

Effects of miRNA-195 on migration and invasion of AMC-HN-8 cells. The potential effects of miRNA-195 on cell migration and invasion were assessed using Transwell 
A

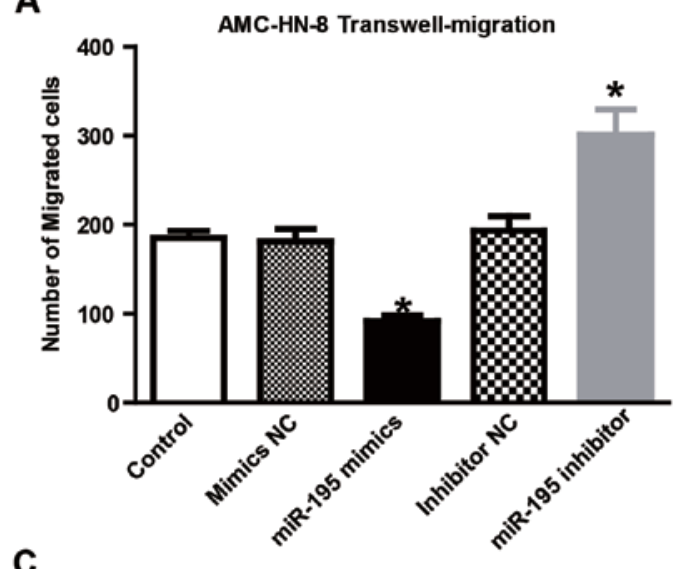

B

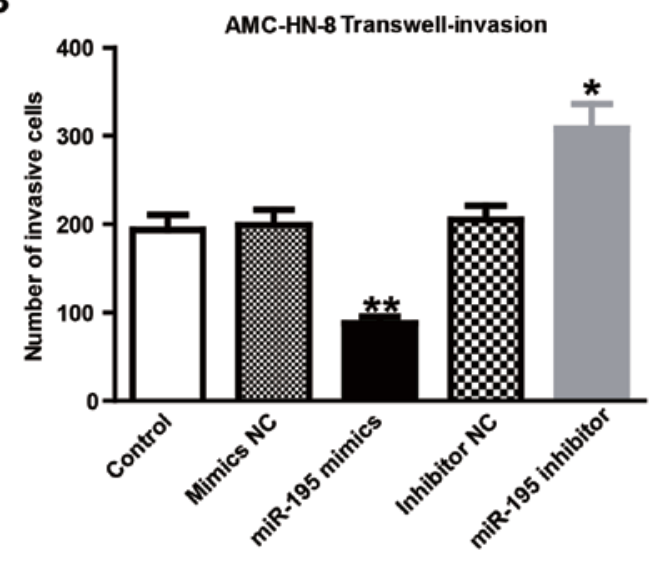

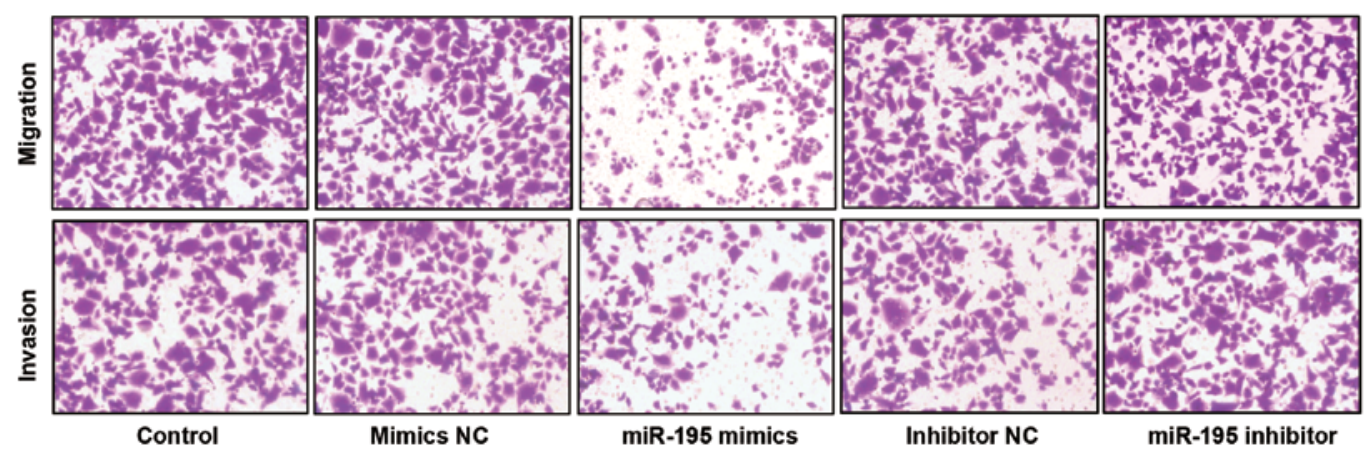

Figure 3. Overexpression of miRNA-195 inhibits the migration and invasion of cultured AMC-HN-8 cells. (A) Number of migrated AMC-HN-8 cells in different treatment groups. (B) Number of invaded AMC-HN-8 cells in different treatment groups. Data are expressed as mean \pm standard error of the mean, $\mathrm{n}=3$. ${ }^{*} \mathrm{P}<0.05,{ }^{* *} \mathrm{P}<0.01$ vs. control group. (C) The AMC-HN-8 cells observed in the lower chambers following migration and invasion assays (original magnification, x200). miRNA/miR, microRNA; NC, negative control.

migration and invasion assays. AMC-HN-8 cells were selected for overexpression and knockdown of miRNA-195 using transient transfection, and Transwell migration and Matrigel invasion assays demonstrated that miRNA-195 overexpression resulted in a significant reduction of AMC-HN-8 cell migration (Fig. $3 \mathrm{~A}$; $\mathrm{P}<0.05$ ) and invasion rate (Fig. 3B; $\mathrm{P}<0.01$ ) compared with the NC group. By contrast, miRNA-195 knockdown resulted in an increase of AMC-HN-8 cell migration (Fig. $3 \mathrm{~A} ; \mathrm{P}<0.05$ ) and invasion rate (Fig. 3B; $\mathrm{P}<0.05$ ) compared with the NC group. Fig. 3C shows the representative pictures of invasion and migration of AMC-HN-8 cells.

Association of VEGFR2/PI3K/AKT signal pathways with the miRNA-195-induced pro-apoptotic effect on AMC-HN-8 cells. The present study investigated whether miRNA-195 produced a pro-apoptotic effect by targeting VEGFR2 and downstream signaling pathway proteins in AMC-HN-8 cells (Fig. 4). As was hypothesized, negative regulation was identified between miRNA-195 and VEGFR2 in AMC-HN-8 cells (Fig. 4D). To evaluate the effect on cell apoptotic protein expression, AMC-HN-8 cells were transiently transfected with miRNA-195 mimics, miRNA-195 inhibitor or their NC. Lower levels of Bcl-2 (Fig. 4B) were detected in the miR-195 mimics-transfected cells compared with cells transfected with the NC or untransfected cells. In addition, higher levels of Bax (Fig. 4A) and caspase-3 (Fig. 4C) were detected compared with cells transfected with $\mathrm{NC}$ or untransfected cells. Additionally, since the AKT/p-AKT is the upstream of Bax, Bcl-2 and caspase-3, the expression levels of AKT/p-AKT were also detected. As shown in Fig. 4E and $\mathrm{F}$, the expression level of $\mathrm{p}-\mathrm{AKT}$, but not AKT, was significantly decreased in the miR-195 mimics-transfected cells compared with cells transfected with the $\mathrm{NC}$ or untransfected cells.

Overexpression of miRNA-195 inhibits viability, migration and invasion via regulating Raf/MEK/ERK and SRC/FAK signal pathways respectively in AMC-HN-8 cells. To evaluate whether overexpression of miRNA-195 serves a role in the regulation of the viability, migration and invasion of AMC-HN-8 cells, AMC-HN-8 cells were transiently transfected with miRNA-195 mimic, miRNA-195 inhibitor or their NC. Among various signaling molecules downstream of VEGFR2, the Raf/MEK/ERK pathway mainly promotes cell growth and differentiation. The western blot analysis data from the present study demonstrated that the RAF, p-MEK and p-ERK genes were significantly inhibited by upregulation of miRNA-195 at the protein level in AMC-HN-8 cells (Fig. 5A; P 0.05 ) compared with the control group. In addition, it was also identified that miRNA-195 overexpression resulted in lower expression of $\mathrm{p}$-SRC and $\mathrm{p}$-FAK proteins in AMC-HN-8 cells (Fig. 5B; $\mathrm{P}<0.05$ ) compared with the control group. Together, these data reveal that overexpression of miRNA-195 is able to inhibit VEGFR2 and downstream signaling pathways, including Raf/MEK/ERK and SRC/FAK, 
A
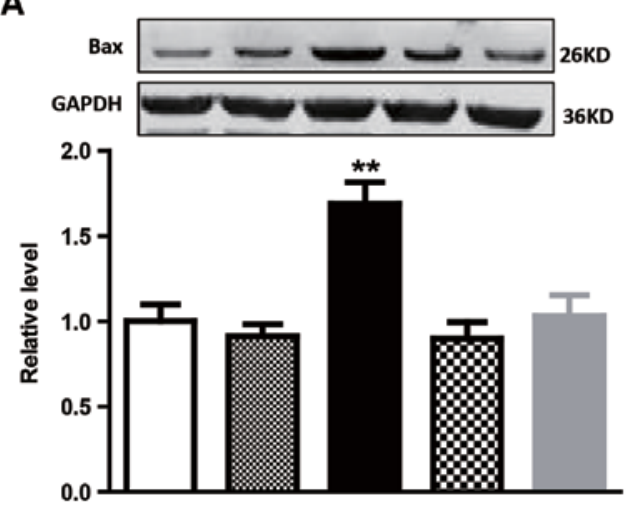

C
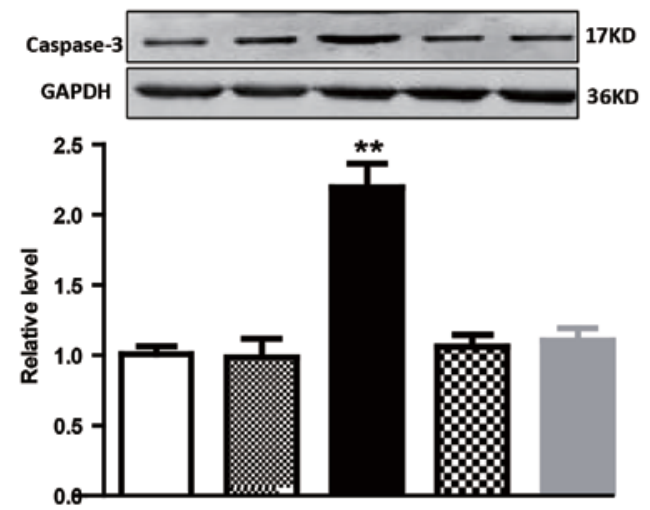

E
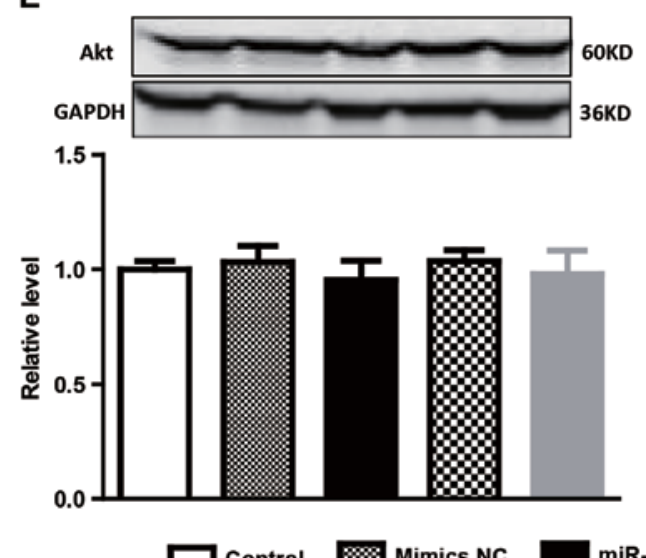

B
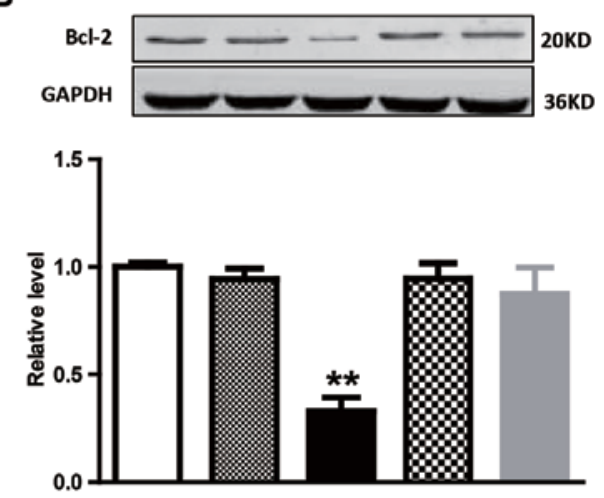

D
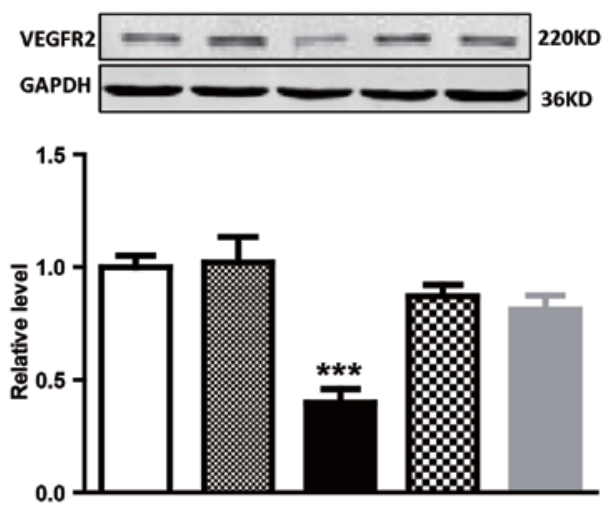

$\mathbf{F}$

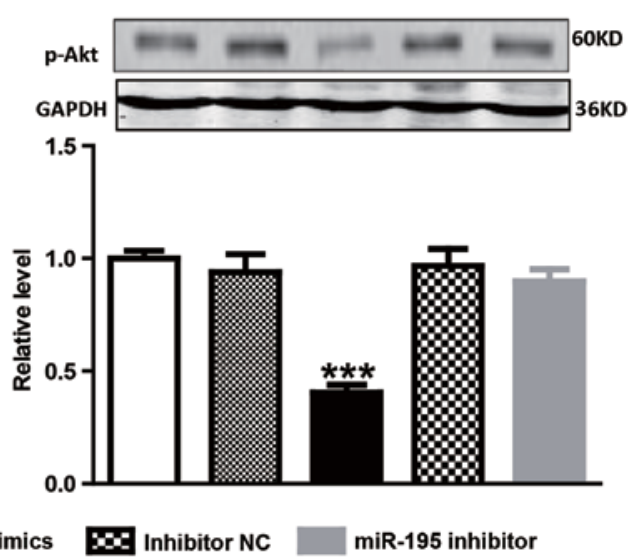

Figure 4. Protein levels in differently treated AMC-HN-8 cells were determined by western blotting. Protein levels of (A) Bax, (B) Bcl-2, (C) caspase-3, (D) VEGFR2, (E) AKT and (F) p-AKT were detected. Data are expressed as mean \pm standard error of the mean, $\mathrm{n}=4 .{ }^{* * *} \mathrm{P}<0.01$ or ${ }^{* * * *} \mathrm{P}<0.001$ vs. control group. VEGFR2, vascular endothelial growth factor receptor 2; AKT, protein kinase B; p, phosphorylated; Bcl-2, B-cell lymphoma 2; Bax, Bcl-2-like protein 4.

in AMC-HN-8 cells, which inhibit LSCC cell viability, migration and invasion, respectively.

\section{Discussion}

The present study identified downregulation of miRNA-195 in LSCC tissue samples compared with matched normal tissue samples and that overexpression of miRNA-195 in AMC-HN-8 cells was able to suppress cell viability, migration and invasion, and induce cell apoptosis. In addition, the data suggest that suppression of VEGFR2 and its downstream signal proteins, including Raf/MEK/ERK1/2, SRC/FAK and apoptosis-related proteins, mediate the antitumor effect of miRNA-195 (Fig. 6). The results of the present study aid the understanding of the mechanisms of miRNA-195 in regulating the viability, migration and apoptosis in LSCC cell lines and support the suggestion that miRNAs may serve as potential therapeutic and drug targets.

With the development of RT-qPCR and microarrays, a number of recent studies $(24,25)$ have identified that dysregulation of miRNAs is closely associated with the development and progression of various types of cancer, via cell viability, cell invasion and apoptosis, by acting as oncogenes or tumor suppressor genes. However, the mechanism of miRNAs in 
A

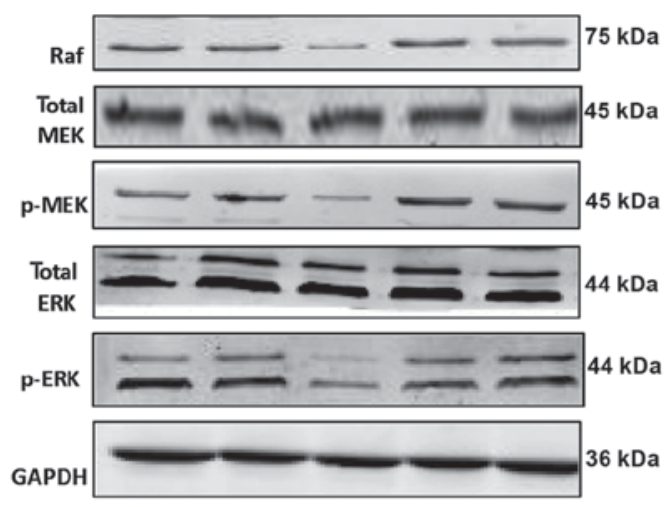

B

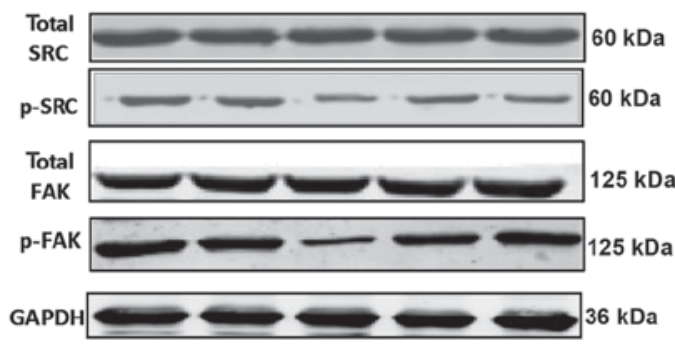

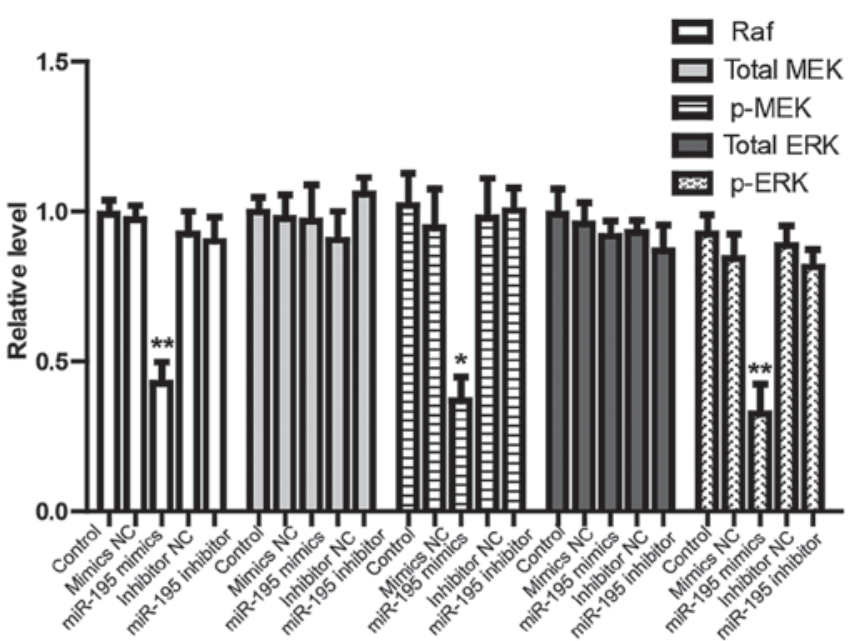

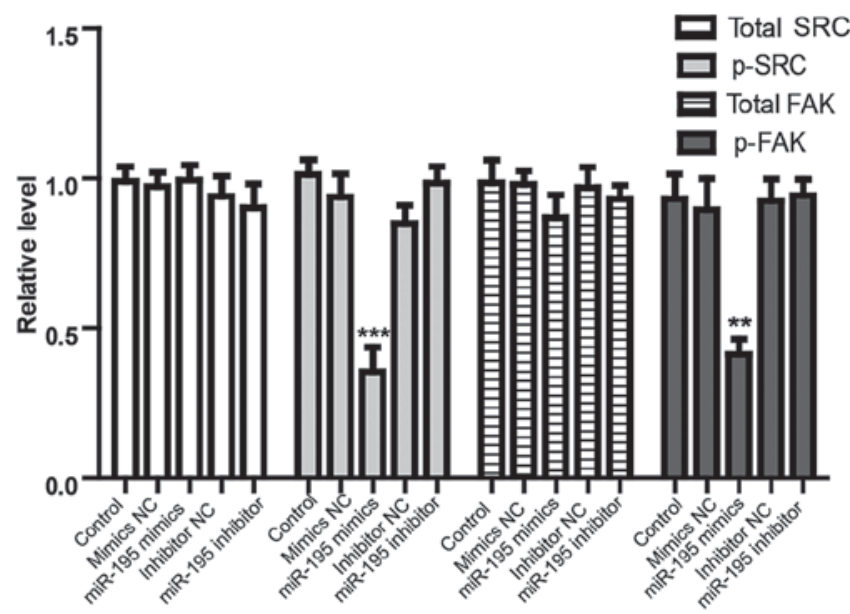

Figure 5. Effects of miRNA-195 overexpression on Raf/MEK/ERK and SRC/FAK signaling pathways in cultured AMC-HN-8 cells. Proteins expression of (A) Raf, total-MEK, p-MEK, total-ERK and p-ERK, and (B) total SRC, p-SRC, total FAK, and p-FAK were determined by western blotting. Data are expressed as mean \pm standard error of the mean, $\mathrm{n}=4 .{ }^{*} \mathrm{P}<0.05,{ }^{* *} \mathrm{P}<0.01$ or ${ }^{* * * *} \mathrm{P}<0.001$ vs. control group. miRNA/miR, microRNA; NC, negative control; MEK, mitogen activated protein kinase kinase; p, phosphorylated; ERK, extracellular signal-regulated kinase; FAK, focal adhesion kinase.

LSCC remains to be elucidated. miRNA-195 downregulation has been reported in various types of cancer, including human hepatocellular carcinoma (26), breast cancer (18), esophageal squamous cell carcinoma (27), adrenocortical cancer (28), colorectal cancer (29) and human tongue squamous cell carcinoma (30). The present study examined the expression level of miRNA-195 in human LSCC tissues using RT-qPCR, which demonstrated that miRNA-195 was downregulated in primary LSCC tissues compared with matched adjacent non-cancerous tissues. These data suggest that downregulation of miRNA-195 in human LSCC tissues may be one of the molecular events causing its development. Ina previous study (31), miRNA-195 was observed to regulate a number of target proteins, which are associated with the cell cycle, apoptosis and viability in multiple diseases, such as cancer, schizophrenia and heart failure (31-33). However, to the best of our knowledge, the expression level of miR-195 in human LSCC, its clinical role and its prognostic value have not been fully investigated thus far. The present study demonstrated the role of miRNA-195 in regulating cell biological functions during the development of LSCC. It was identified that overexpression of miRNA-195 significantly inhibited cell viability, migration and invasion, and promoted apoptosis in AMC-HN-8 LSCC cells. The identification of miRNA-195 target genesis is significant for the improved understanding of the role of miRNA-195 in tumorigenesis. At present, certain genes such as WEE1 (34), cyclin dependent kinase 6 (35), and $\mathrm{Bcl}-2$ have been confirmed as targets of the miRNA-195 gene. According to the expression level ofmiRNA-195 in LSCC, the present study selected the presumed tumor-related gene VEGFR2 as a potential target of miRNA-195 among the predicted genes. The current study identified that overexpression of miRNA-195 represses the expression level of VEGFR2 in AMC-HN-8 cells. VEGFR2 mediates a variety of important molecular signaling pathways via its downstream signal proteins, including Raf/MEK/ERK1/2, SRC/FAK and apoptosis-related proteins, which serve a key role in regulating tumor development (36). Data from the present study further support the hypothesis that inhibition of VEGFR2/Raf/MEK/ERK and VEGFR2/SRC/FAK pathways regulates cancer cell growth and migration, respectively, mediated by miRNA-195 upregulation in vitro. It is noteworthy that not only was the expression of VEGFR2 in AMC-HN-8 cells treated with miRNA-195 mimics significantly lower than the control group, but also that 


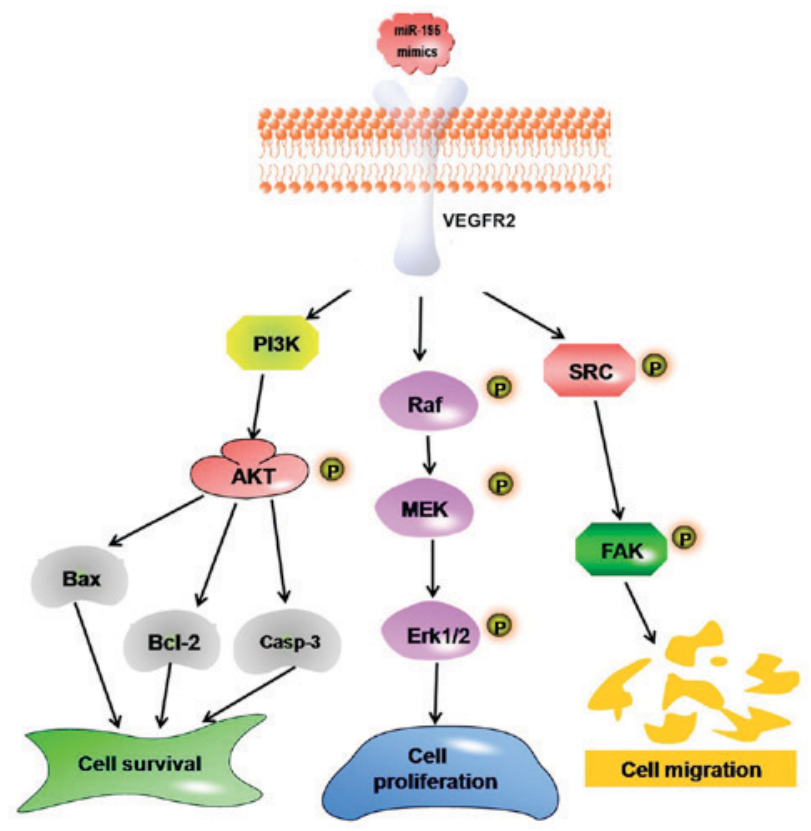

Figure 6. Schematic illustration of the possible targeting and signaling mechanisms by which miRNA-195 produces antitumor effects in cultured AMC-HN-8 cells. Increased miRNA-195 represses cells viability, migration and invasion by targeting VEGFR2, which leads to decreases in the activation of the Raf/MEK/ERK and SRC/FAK signaling pathways in cultured AMC-HN-8 cells. In addition, miRNA-195 may induce AMC-HN-8 cell apoptosis by downregulating the PI3K/AKT signaling pathway. miRNA/miR, microRNA; VEGFR2, vascular endothelial growth factor receptor 2; PI3K, phosphoinositide 3-kinase; AKT, protein kinase B; Bcl-2, B-cell lymphoma 2; Casp-3, caspase-3; MEK, mitogen activated protein kinase kinase; ERK, extracellular signal-regulated kinase; FAK, focal adhesion kinase; $\mathrm{p}$, phosphorylated.

the levels of p-AKT and Bcl-2 in LSCC cells with miRNA-195 overexpression were significantly decreased. These results suggest that the miRNA-195/VEGFR2 signaling pathway may serve an important role in the development of LSCC. However, it should be noted that the present study was performed in cell lines and the findings may not be capable of being extrapolated directly to animal models or to humans. There is a need for caution when applying the results of the present study to animals or patients.

The present study identified a novel antitumor miRNA, miRNA-195, in human LSCC tissue. The data suggest that the therapeutic potential of miRNA-195 in modulating cell growth, migration and apoptosis during the pathophysiological progression of LSCC, and the underlying mechanism, is associated with the inhibition of VEGFR2 and downstream signaling pathways, including Raf/MEK/ERK, SRC/FAK and PI3K/AKT. The present study indicates that exogenous application of miRNA-195 may be a promising intervention in the management of human LSCC and the associated pathological processes. The findings provide rationale for additional studies to investigate whether the miR-195 mechanisms associated with modulating cell growth, migration and apoptosis also operate in the clinical setting.

\section{Acknowledgements}

The authors thank all members of their laboratory for helpful discussions and comments on the present study.

\section{References}

1. Siegel RL, Fedewa SA, Miller KD, Goding-Sauer A, Pinheiro PS, Martinez-Tyson D and Jemal A: Cancer statistics for hispanics/latinos, 2015. CA Cancer J Clin 65: 457-480, 2015.

2. McGuire S: World cancer report 2014. Geneva, Switzerland: World Health Organization, International Agency for Research on Cancer, WHO Press, 2015. Adv Nutr 7: 418-419, 2016.

3. Torre LA, Bray F, Siegel RL, Ferlay J, Lortet-Tieulent J and Jemal A: Global cancer statistics, 2012. CA Cancer J Clin 65: 87-108, 2015

4. Li F, Liu Y, Kan X, Li Y, Liu M and Lu JG: Elevated expression of integrin alphav and $\beta 5$ subunit in laryngeal squamous-cell carcinoma associated with lymphatic metastasis and angiogenesis. Pathol Res Pract 209: 105-109, 2013.

5. Canis M, Ihler F, Martin A, Wolff HA, Matthias C and Steiner W: Results of 226 patients with T3 laryngeal carcinoma after treatment with transoral laser microsurgery. Head Neck 36: 652-659, 2014.

6. Yu ST, Zhou Z, Cai Q, Liang F, Han P, Chen R and Huang XM: Prognostic value of the $\mathrm{C}$-reactive protein/albumin ratio in patients with laryngeal squamous cell carcinoma. Onco Targets Ther 10: 879-884, 2017.

7. Liu JC, Shen WC, Shih TC, Tsai CW, Chang WS, Cho Y, Tsai CH and Bau DT: The current progress and future prospects of personalized radiogenomic cancer study. Biomedicine (Taipei) 5: 2,2015 .

8. Lee Y, Ahn C, Han J, Choi H, Kim J, Yim J, Lee J, Provost P, Rådmark O, Kim S and Kim VN: The nuclear RNase III Drosha initiates microRNA processing. Nature 425: 415-419, 2003.

9. Tutar L, Tutar E and Tutar Y: MicroRNAs and cancer; an overview. Curr Pharm Biotechnol 15: 430-437, 2014.

10. Qiu F, Yang L, Ling X, Yang R, Yang X, Zhang L, Fang W, Xie C, Huang D, Zhou Y and Lu J: Sequence variation in mature microRNA-499 confers unfavorable prognosis of lung cancer patients treated with platinum-based chemotherapy. Clin Cancer Res 21: 1602-1613, 2015.

11. Lv G,Hu Z, Tie Y, Du J, Fu H, Gao X and Zheng X: MicroRNA-451 regulates activating transcription factor 2 expression and inhibits liver cancer cell migration. Oncol Rep 32: 1021-1028, 2014.

12. Ribeiro-dos-Santos Â, Khayat AS, Silva A, Alencar DO, Lobato J, Luz L, Pinheiro DG, Varuzza L, Assumpção M, Assumpção P, et al: Ultra-deep sequencing reveals the microRNA expression pattern of the human stomach. PLoS One 5: e13205, 2010.

13. Brockway S and Zeleznik-Le NJ: WEE1 is a validated target of the microRNA miR-17-92 cluster in leukemia. Cancer Genet 208: 279-287, 2015.

14. Kim J, Jeong D, Nam J, Aung TN Gim JA, Park KU and Kim SW: MicroRNA-124 regulates glucocorticoid sensitivity by targeting phosphodiesterase 4B in diffuse large B cell lymphoma. Gene 558: 173-180, 2015.

15. Zhao D, Tu Y, Wan L, Bu L, Huang T, Sun X, Wang K and Shen B: In vivo monitoring of angiogenesis inhibition via down-regulation of mir-21 in a VEGFR2-luc murine breast cancer model using bioluminescent imaging. PLoS One 8: e71472, 2013.

16. Vishnubalaji R, Hamam R, Abdulla MH, Mohammed MA, Kassem M, Al-Obeed O, Aldahmash A and Alajez NM: Genome-wide mRNA and miRNA expression profiling reveal multiple regulatory networks in colorectal cancer. Cell Death Dis 6: e1614, 2015.

17. Zhang S, Hu F, Liang H, Liu Y, Yang J and Zhou W: Association between a miRNA-146a polymorphism and susceptibility to head and neck squamous cell carcinoma in Chinese patients: A meta-analysis of 8 case-control studies. PLoS One 12: e0186609, 2017.

18. Heneghan HM, Miller N, Kelly R, Newell J and Kerin MJ: Systemic miRNA-195 differentiates breast cancer from other malignancies and is a potential biomarker for detecting noninvasive and early stage disease. Oncologist 15: 673-682, 2010.

19. Itesako T, Seki N, Yoshino H, Chiyomaru T, Yamasaki T, Hidaka H, Yonezawa T, Nohata N, Kinoshita T, Nakagawa M and Enokida $\mathrm{H}$ : The microRNA expression signature of bladder cancer by deep sequencing: The functional significance of the miR-195/497 cluster. PLoS One 9: e84311, 2014.

20. Collier R: World medical association updates ethical code for physicians. CMAJ 189: E1372, 2017. 
21. Livak KJ and Schmittgen TD: Analysis of relative gene expression data using real-time quantitative PCR and the 2(-Delta Delta C(T)) method. Methods 25: 402-408, 2001

22. Tu Y, Wan L, Fan Y, Wang K, Bu L, Huang T, Cheng Z and Shen B: Ischemic postconditioning-mediated miRNA-21 protects against cardiac ischemia/reperfusion injury via PTEN/Akt pathway. PLoS One 8: e75872, 2013

23. Justus CR, Leffler N, Ruiz-Echevarria M and Yang LV: In vitro cell migration and invasion assays. J Vis Exp: Jun 1, 2014 doi: $10.3791 / 51046$.

24. Galoian KA, Guettouche T, Issac B, Qureshi A and Temple HT: Regulation of onco and tumor suppressor MiRNAs by mTORC1 inhibitor PRP-1 in human chondrosarcoma. Tumour Biol 35 2335-2341, 2014.

25. Wang H: Predicting cancer-related MiRNAs using expression profiles in tumor tissue. Curr Pharm Biotechnol 15: 438-444, 2014.

26. Yang X, Yin J, Yu J, Xiang Q, Liu Y, Tang S, Liao D, Zhu B, $\mathrm{Zu}$ X, Tang H and Lei X: miRNA-195 sensitizes human hepatocellular carcinoma cells to 5-FU by targeting BCL-w. Oncol Rep 27: 250-257, 2012.

27. Fu MG, Li S, Yu TT, Qian LJ, Cao RS, Zhu H, Xiao B, Jiao CH, Tang NN, Ma JJ, et al: Differential expression of miR-195 in esophageal squamous cell carcinoma and miR-195 expression inhibits tumor cell proliferation and invasion by targeting of Cdc42. FEBS Lett 587: 3471-3479, 2013.

28. Chabre O, Libé R, Assie G, Barreau O, Bertherat J, Bertagna X, Feige JJ and Cherradi N: Serum miR-483-5p and miR-195 are predictive of recurrence risk in adrenocortical cancer patients Endocr Relat Cancer 20: 579-594, 2013
29. Wang X, Wang J, Ma H, Zhang J and Zhou X: Downregulation of miR-195 correlates with lymph node metastasis and poor prognosis in colorectal cancer. Med Oncol 29: 919-927, 2012.

30. Jia LF, Wei SB, Gong K, Gan YH and Yu GY: Prognostic implications of micoRNA miR-195 expression in human tongue squamous cell carcinoma. PLoS One 8: e56634, 2013.

31. He JF, Luo YM, Wan XH and Jiang D: Biogenesis of MiRNA-195 and its role in biogenesis, the cell cycle, and apoptosis. J Biochem Mol Toxicol 25: 404-408, 2011.

32. Shi W, Du J, Qi Y, Liang G, Wang T, Li S, Xie S, Zeshan B and Xiao Z: Aberrant expression of serum miRNAs in schizophrenia. J Psychiatr Res 46: 198-204, 2012.

33. He X, Ji J, Wang T, Wang MB and Chen XL: Upregulation of circulating miR-195-3p in heart failure. Cardiology 138: 107-114, 2017.

34. Bhattacharya A, Schmitz U, Wolkenhauer O, Schonherr M, Raatz Y and Kunz M: Regulation of cell cycle checkpoint kinase WEE1 by miR-195 in malignant melanoma. Oncogene 32: 3175-3183, 2013.

35. Deng H, Guo Y, Song H, Xiao B, Sun W, Liu Z, Yu X, Xia T, Cui L and Guo J: MicroRNA-195 and microRNA-378 mediate tumor growth suppression by epigenetical regulation in gastric cancer. Gene 518: 351-359, 2013

36. Sun P, Wang L, Lu Y, Liu Y, Li L, Yin L, Zhang C, Zhao W, Shen B and $\mathrm{Xu}$ W: MicroRNA-195 targets VEGFR2 and has a tumor suppressive role in ACHN cells via PI3K/Akt and Raf/MEK/ERK signaling pathways. Int J Oncol 49: 1155-1163, 2016. 